\title{
Impactos sobre a conservação de recursos naturais em áreas de exploração mineral
}

\section{Fredson Pereira da Silva ${ }^{1}$ e Carlos Alberto Batista Santos ${ }^{2}$}

${ }^{1}$ Universidade Estadual do Ceará. Programa de Pós-Graduação em Geografia. Av. Dr. Silas Munguba, 1700. Campus do Itaperi. Fortaleza-CE, Brasil (CEP 60714-903).

${ }^{2}$ Universidade do Estado da Bahia. Departamento de Tecnologia e Ciências Sociais. Programa de Pós-Graduação em Ecologia Humana e Gestão Socioambiental. Av. Edgard Chastinet Guimarães, S/№. São Geraldo. Juazeiro-BA, Brasil (CEP 48904-711). E-mail: cabsantos@uneb.br.

Resumo. As questões que envolvem a conservação da diversidade de seres vivos no mundo vem sendo abordados frequentemente em todos os níveis de organização social, entre os temas está a extinção das espécies, como consequência das agressões ao meio ambiente, ás áreas de exploração mineral, são áreas susceptíveis a causarem dados não só ao solo, mas também a toda a diversidade de vida no local, com repercussões no seu entorno, que provoca redução ou destruição de hábitat, afugentamento da fauna, promovendo a morte de espécies da fauna e da flora terrestres e aquáticas. 0 objetivo deste estudo é apresentar uma lista de espécies da fauna e flora encontradas na Região da Vila Renascer, Petrolina, Estado de Pernambuco, e discutir os impactos na região, que ameaçam essa diversidade. Este estudo foi realizado a partir de entrevistas com a população local residente no entorno de uma mineradora de gnaisse, acerca dos impactos e importância da mineração. A amostragem foi aleatória, sendo selecionados 87 informantes, como critério para inclusão dos participantes na pesquisa foi adotado a idade $>18$ anos e escolaridade, optando-se pelos alfabetizados. Os resultados registra que a exploração da rocha, é apontada pelos moradores como redução de espécies da fauna está o Galea spixii, o tatu Euphractus sexcinctus e cascavel Crotalus durissus terrificus pelas mudanças climáticas e pela atividade de caça desses animais e da flora Caesalpinia pyramidalis e o Spondias tuberosa devido ao desmatamento para retirada da rocha, queimadas e abertura de novas vias de acesso para mineração. Diante do exposto, a paisagem como também toda a dinâmica do meio ambiente é alterada, levando extinção de espécies da caatinga, transtornos na saúde da população e também depredação do patrimônio particular, diante disso, não se vislumbra compatibilidade entre a extração de minérios e os objetivos de proteção da fauna e da flora.
Recebido

$19 / 08 / 2020$

Aceito

$06 / 12 / 2020$

Disponível on line

$07 / 12 / 2020$

Publicado

$31 / 12 / 2020$

Acesso aberto

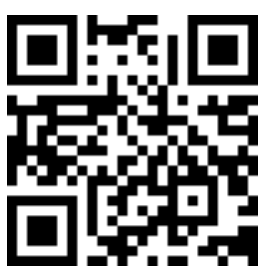

ORCID

(D) 0000-0003-1363-948X

Fredson Pereira da

Silva

0000-0002-2049-5237

Carlos Alberto Batista

Santos 
Palavras-chave: Semiárido; Caatinga; Mineração; Gnaisse; Espécies.

\begin{abstract}
Impacts on the conservation of natural resources in mineral exploration areas. The issues involving the conservation of the diversity of living beings in the world have been addressed frequently at all levels of social organization, among the issues is the extinction of species, as a consequence of aggressions to the environment, the areas of mineral exploration, are areas that can cause not only the soil, but also all the diversity of life in the place, with repercussions on their surroundings. This causes habitat reduction or destruction, scaring away fauna, promoting the death of terrestrial and aquatic fauna and flora species. The objective of this study is to present a list of species of fauna and flora found in the region of Vila Renascer, Petrolina, Pernambuco State, Northeast Brazil, and discuss the impacts on the region, which threaten this diversity. This study was carried out from interviews with the local population living around a gneiss mining company, about the impacts and importance of mining. The sample was random, being selected 87 informants, as criteria for inclusion of the participants in the research was adopted the age $>18$ years and schooling, opting for the literate ones. The results show that the exploration of the rock, is indicated by the residents as a reduction of species of fauna is the Spix's yellow-toothed cavy Galea spixii, the armadillo Euphractus sexcinctus and rattlesnake Crotalus durissus terrificus by climate change and hunting activity of these animals and the flora Caesalpinia pyramidalis and the Spondias tuberosa due to deforestation to remove the rock, burning and opening of new access routes for mining. In view of the above, the landscape as well as the entire dynamics of the environment is altered, leading to the extinction of species of the Caatinga, disorders in the health of the population and also depredation of private patrimony. In view of this, there is no prospect of compatibility between the extraction of minerals and the objectives of protection of fauna and flora.
\end{abstract}

Keywords: Semiarid; Caatinga; Mining; Gnaisse; Species.

\title{
Introdução
}

As questões que envolvem a conservação da diversidade de seres vivos no mundo, envolvem temas que vem sendo abordados frequentemente em todos os níveis de organização social. Essa diversidade é definida como a variabilidade dos organismos vivos de qualquer origem, compreendendo os ecossistemas dos quais eles fazem parte, seja estes terrestres, aquáticos e os complexos ecológicos (Lèvêque, 1999).

Entre os assuntos mais discutidos e relacionados com a biodiversidade, está a extinção das espécies, como consequência das agressões ao meio ambiente (Ross, 2012).

Para regulamentar e propor diretrizes para conservação da biodiversidade e minimizar os danos causados, em 1981, foi criada a Política Nacional de Meio Ambiente (PNMA), por meio da Lei no 6.938 (Brasil, 1981). Em 1985, surgiu o Ministério do Meio 
Ambiente (MMA) e, finalmente, em 1988 foi publicada a Constituição Federal (Brasil, 1988) e a Lei de Crimes Ambientais (Brasil, 1998) (Peccatiello, 2011).

A partir da promulgação da Constituição de 1988, no art. 24 destaca que a preservação/conservação da fauna e da flora passavam então a ser competência comum da União, dos Estados, do Distrito Federal e dos municípios, em legislar concorrentemente sobre florestas, caça, pesca, fauna, conservação da natureza, defesa do solo e dos recursos naturais, proteção do meio ambiente e controle da poluição (Drummond et al., 2005) e afirmando no art. 225, que "todos têm o direito ao meio ambiente ecologicamente equilibrado, bem de uso comum do povo e essencial à sadia qualidade de vida, impondo-se ao Poder Público e à coletividade o dever de defendê-lo e preservá-lo para as presentes e futuras gerações" (Brasil, 1988).

As áreas de exploração mineral, são áreas susceptíveis a causarem dados não só ao solo, mas também a toda a diversidade de vida no local, com repercussões no seu entorno. Este artigo apresenta uma lista de espécies da fauna e flora encontradas na região da Vila Renascer, Petrolina, Estado de Pernambuco, e discute os impactos na região, que ameaçam essa diversidade.

\section{Impactos ambientais gerados pela exploração mineral}

Os impactos da mineração variam conforme a substância explorada, o método de lavra e as características naturais e humanas locais (Viana, 2015). Os efeitos no ambiente são relativos a todas as fases da exploração, desde a abertura de cava até o beneficiamento do minério (Bacci et al., 2006), interferindo nos diferentes meios, físico (solo e recursos hídricos), biótico (fauna e flora) e socioeconômico (Pereira et al., 2014).

Estes impactos podem ter efeitos danosos no equilíbrio dos ecossistemas, tais como a redução ou destruição de hábitat, afugentamento da fauna, promovendo a morte de espécies da fauna e da flora terrestres e aquáticas, incluindo eventuais espécies em extinção, promovendo como consequência a interrupção de corredores de fluxos gênicos e de movimentação da biota.

Em relação ao meio antrópico, a mineração pode causar não apenas o desconforto ambiental, mas também impactos à saúde causados pela poluição sonora, do ar, da água e do solo.

A desfiguração da paisagem é outro aspecto gerado pela mineração cujo impacto depende do volume de escavação e da visibilidade em razão de sua localização (Mechi e Sanches, 2010).

No âmbito internacional uma pesquisa em área Semiárida da Mongólia interior da China, revelou os impactos na paisagem pela exploração da mineração a céu aberto, como também do carvão mineral no subsolo, num raio de $5 \mathrm{~km}$ em torno da mineração na Cidade de Xilinhot, como também a ecologia da paisagem por meio de perturbações e a deterioração do ecossistema (Wu et al., 2020).

Wahsha et al. (2019) em estudo em Belluno, Região Nordeste da Itália, numa área de mineração de mercúrio, constatou a acidificação da água e a contaminação de solos e plantas por ferro e mercúrio e pelos restos de resíduos descartados depois da exploração a céu aberto.

No âmbito nacional os estudos relacionados ao impacto da mineração sobre a biodiversidade ainda são incipientes. Pena et al. (2017) em estudo realizado em áreas de mineração verificaram os impactos sobre espécies de anuros e aves endêmicas dos montes do leste do Brasil, a mineração afetou cerca de 40\% das espécies, destruindo, fragmentando e degradando habitats naturais, liberando resíduos tóxicos e alterando a dinâmica do uso da terra nas regiões mineradas, afetando espécies, como por exemplo a rã Bokermannohyla sagarana Leite, Pezzuti e Drummond, 2011 e o beija flor de gravata verde Augastes scutatus Temminck, 1822. 
Rudke et al. (2020) em investigação na Amazônia, no Estado de Rondônia identificou mais de 500 processos de exploração de minerais como a cassiterita e ouro em Unidades de Conservação, muitas delas na margem do Rio Madeira, afetando a área de proteção permanente (APP), destacando como a mineração afetou a área da Floresta Nacional do Jamari, onde se encontra uma das espécies da fauna mais ameaçada da região, a onça-pintada Panthera onca Linnaeus, 1758, símbolo brasileiro da conservação da biodiversidade.

Pires Junior (2019), em estudo realizado no Município de São Sebastião da Vargem Alegre, Minas Gerais, demonstra como os impactos gerados pelo desmatamento para exploração do minério bauxita na Mata Atlântica, cooperam na abertura de fragmentos florestais que podem prejudicar populações de espécies da herpetofauna. 0 autor registrou a rã Ischnocnema verrucosa, Reinhardt e Lütken, 1862 e a rã Zachaenus carvalhoi Izecksohn, 1983, duas espécies que não possuem dados de status ecológico bem definidos, e Myersiella micros (Duméril e Bibron, 1841), Haddadus binotatus (Spix, 1824), Adenomera marmorata (Steindachner, 1867) e Ecpleopus gaudichaudii (Duméril e Bibron, 1839), que precisam da vegetação para seu desenvolvimento.

Pacheco e Santos (2019) em investigação na Comunidade Rural de Paredão, no Município de Juazeiro, Bahia, identificaram impactos no Bioma Caatinga no semiárido nordestino, pela exploração da mineração da malaquite e cobre, com derrubada de espécies da flora como o umbuzeiro Spondias tuberosa Arr. Cam. e erosão no solo e subsolo, deixando exposto aos processos erosivos, podendo causar o processo de desertificação, que afeta diretamente a fauna local.
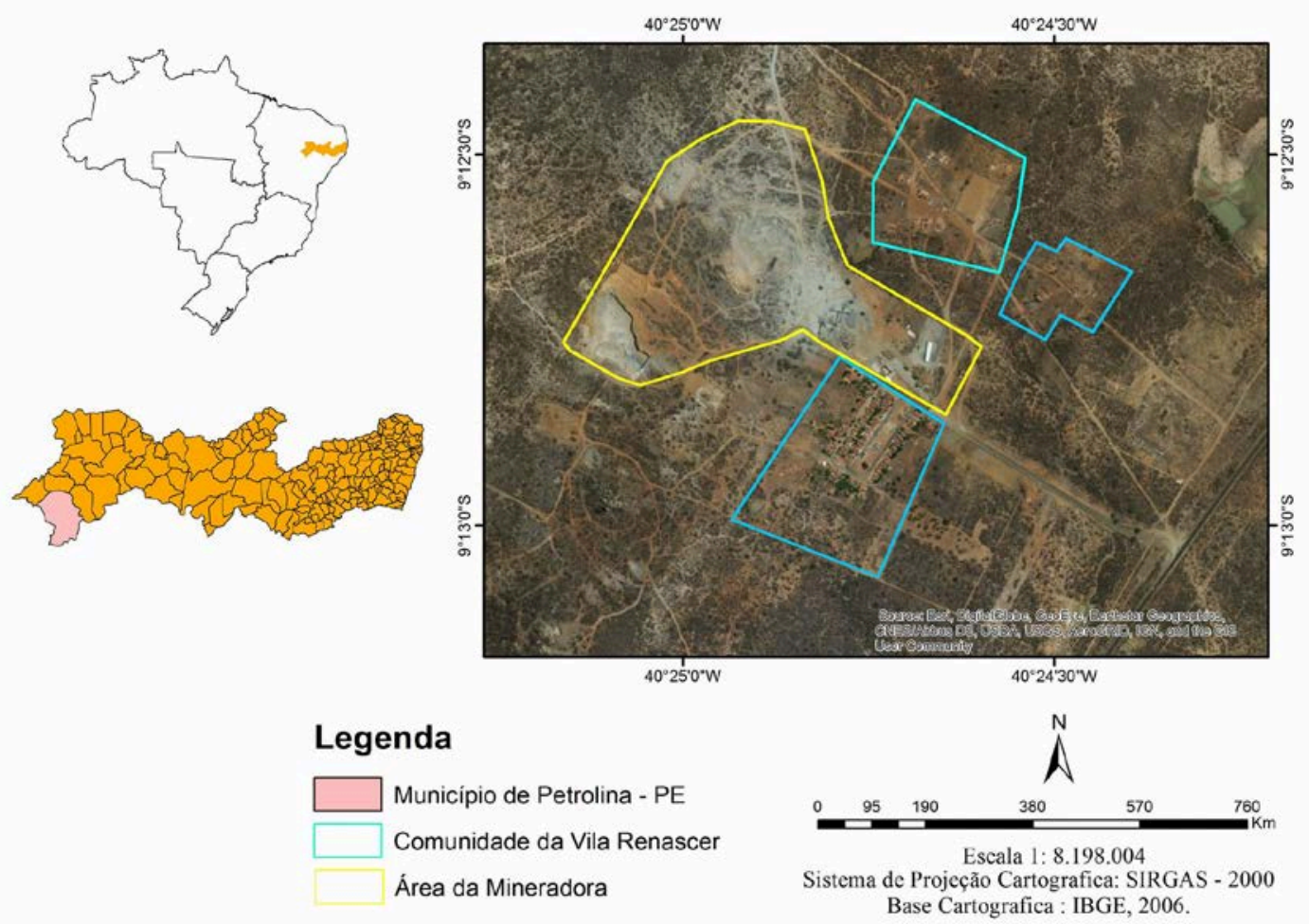

Figura 1. Área da Comunidade da Vila Renascer e da mineração do gnaisse em Petrolina, Pernambuco Nordeste do Brasil. Fonte: Silva et al. (2018b). 


\section{Material e métodos}

A Vila Renascer, comunidade da Zona Rural está localizada no Município de Petrolina, Pernambuco, Nordeste do Brasil (Figura 1). Está posicionada entre inselbergs, peculiares da região semiárida com rocha do tipo gnaisse, com solos do tipo planossolo e neossolo litólico, a vegetação é do tipo caatinga arbustiva densa e esparsa. A comunidade tem quarenta anos de existência, sendo constituída por 300 habitantes e cerca de 150 casas. 0 perfil socioeconômico da comunidade é baixo, com renda de até um salário mínimo, fruto de atividades agrícolas que garantia o sustento da população da Vila (Silva et al., 2018b). Este estudo foi aprovado pelo Comitê de Ética da Universidade do Estado da Bahia, parecer 2.023.539.

Os dados foram coletados a partir de entrevistas, com a população residente no entorno de uma mineradora de gnaisse, acerca dos impactos e importância da mineração. As entrevistas foram conduzidas, com a aplicação de questionários semiestruturados (Albuquerque et al., 2014; Santos et al., 2016). A amostragem foi aleatória, sendo selecionados 87 informantes, o que corresponde a $29 \%$ da população total (300 habitantes). Como critério para inclusão dos participantes na pesquisa foi adotado a idade $>18$ anos e escolaridade, optando-se pelos alfabetizados.

\section{Resultados e discussão}

A extração artesanal de rocha para pavimentação, o paralelepípedo, ganhou destaque a partir do ano de 1980, quando passa a fazer parte da economia local, como fonte de renda para os agricultores locais garantindo recursos para a compra de mantimentos (Silva et al., 2018b).

As taxas de extinção estão destinadas a aumentar significativamente, em um futuro breve, caso as medidas preventivas não sejam realizadas urgentemente, de $5 \%$ a $20 \%$ das espécies de fauna e flora estarão ameaçadas de extinção no planeta (Terborgh e Schaik, 2002).

A exploração da rocha, é apontada pelos moradores como redução de espécies, estudos anteriores também demonstram que detonação das rochas nos inselbergs, em áreas de caatinga, promovem a redução da biodiversidade faunística por conta do barulho e das explosões (Pontes et al., 2013; Alves et al., 2008). As espécies animais e vegetais citadas pelos informantes estão elencadas nas Tabelas 1 e 2 .

Os informantes apontam também que o desaparecimento de espécies como preá Galea spixii Wagler, 1831, do tatu peba Euphractus sexcinctus Linnaeus, 1758 e da cascavel Crotalus durissus terrificus Lineu, 1758, são causadas pela seca e pela caça desses animais. Alves et al. (2012), a caça representa uma forma tradicional de manejo da fauna, tendo o papel socioeconômico de fornecer carne às famílias locais. Além disso, no período de seca alguns habitats podem ser alterados, e as espécies migram, buscando garantir sua sobrevivência (Oliveira et al., 2003).

Em relação ao conhecimento das espécies vegetais nas áreas adjacentes da comunidade, as mais citadas foram à catingueira Caesalpinia pyramidalis e o umbuzeiro Spondias tuberosa, ambas com 13 citações, seguidas da jurema Mimosa tenuiflora, com 12 citações.

Observa-se que antes da implantação da mineradora existia uma vegetação de caatinga densa, com maior abundância de espécies, o desmatamento para retirada da rocha, queimadas e abertura de novas vias de acesso são apontadas como as principais causas do desaparecimento das espécies da flora local devido a retirada da mesma para deposição dos materiais explorados, sendo mais afetadas a jurema, catingueira, umbuzeiro e baraúna (Tabela 2) (Silva et al., 2018a), constatação encontrada também em estudos anteriores (Faleiro e Lopes, 2010; Pontes et al., 2013).

Rev. Bras. Gest. Amb. Sustent., 2020, vol. 7, n. 17, p. 1471-1482. 
Tabela 1. Lista de espécies da fauna para a área de mineração do gnaisse na Comunidade Vila Renascer em Petrolina, Pernambuco Nordeste do Brasil.

\begin{tabular}{|c|c|c|c|}
\hline Táxon & Nome popular & $\begin{array}{l}\text { Endemismo } \\
\text { na caatinga } \\
\end{array}$ & $\begin{array}{c}\text { Status de } \\
\text { conservação }\end{array}$ \\
\hline \multicolumn{4}{|l|}{ Répteis } \\
\hline Salvator merianae (Duméril e Bibron, 1839) & Teiú & - & LC \\
\hline Ameiva ameiva (Linnaeus, 1758) & Calango, Bico-doce & - & LC \\
\hline Crotalus durissus (Linnaeus, 1758) & Cascavel & - & LC \\
\hline Micrurus ibiboboca (Merrem, 1820) & Coral verdadeira & - & NA \\
\hline \multicolumn{4}{|l|}{ Aves } \\
\hline Eupsittula cactorum (Kuhl, 1820) & $\begin{array}{l}\text { Periquito da } \\
\text { Caatinga }\end{array}$ & $\mathrm{X}$ & $\mathrm{LC}$ \\
\hline Columbina talpacoti (Temminck, 1810) & Rolinha-roxa & - & $\mathrm{LC}$ \\
\hline Columbina picui (Temminck, 1813) & Rolinha-picui & - & LC \\
\hline Pseudoseisura cristata (Spix, 1824) & Casaca de couro & $\mathrm{X}$ & LC \\
\hline Cyanocorax cyanopogon (Wied 1821) & Cancã & $\mathrm{X}$ & $\mathrm{LC}$ \\
\hline Icterus jamacaii (Gmelin, 1788) & Sofrê & $\mathrm{X}$ & LC \\
\hline Paroaria dominicana (Linnaeus, 1758) & $\begin{array}{c}\text { Cardeal do } \\
\text { nordeste }\end{array}$ & $\mathrm{X}$ & $\mathrm{LC}$ \\
\hline Sporophila albogularis (Cabanis,1847) & Coleirinha & $\mathrm{X}$ & $\mathrm{LC}$ \\
\hline Athene cunicularia (Molina, 1782) & Coruja-buraqueira & - & $\mathrm{LC}$ \\
\hline \multicolumn{4}{|l|}{ Mamíferos } \\
\hline Didelphis albiventris (Lund, 1840) & Saruê & - & $\mathrm{LC}$ \\
\hline Euphractus sexcinctus Linnaeus, 1758 & Tatu peba & - & LC \\
\hline Galea spixii Wagler, 1831 & Préa & - & $\mathrm{LC}$ \\
\hline Kerodon rupestris rupestris (Wied, 1820) & Mocó & $\mathrm{X}$ & VU \\
\hline Dasyprocta prymnolopha (Wagler, 1831) & Cutia & - & $\mathrm{LC}$ \\
\hline Wiedomys pyrrhorhinos (Wied-Neuwied, 1821) & $\begin{array}{c}\text { Rato-do-nariz- } \\
\text { laranja }\end{array}$ & $\mathrm{X}$ & $\mathrm{LC}$ \\
\hline Tamandua tetradactyla (Linnaeus, 1758) & Tamanduá-mirim & - & $\mathrm{LC}$ \\
\hline Cerdocyon thous (Linnaeus, 1766) & Raposa & - & $\mathrm{LC}$ \\
\hline Conepatus semistriatus (Boddaert, 1785) & Gambá-verdadeiro & - & $\mathrm{LC}$ \\
\hline Callithrix penicillata (É. Geoffroy, 1812) & $\begin{array}{c}\text { Sagui-de-tufos- } \\
\text { pretos } \\
\end{array}$ & - & $\mathrm{LC}$ \\
\hline Pecari tajacu (Linnaeus, 1758) & Caititu & - & LC \\
\hline
\end{tabular}

Observação: Status de conservação de acordo com ICMBio (2018). Legenda: NA - Não avaliada; LC Pouco Preocupante; VU - Vulnerável.

Ressalta-se que o Projeto de Lei no 3.458-B (Brasil, 2004), que dispõe sobre a proibição da derrubada do umbuzeiro em todo país, recebeu pareceres da Comissão de Meio Ambiente e Desenvolvimento Sustentável, da Comissão de Meio Ambiente e Desenvolvimento Sustentável e da Comissão de Finanças e Tributação da Câmara dos Deputados, sendo aprovado, e tendo no seu Art. 7ํ, a disposição de que compete ao Poder Público promover processo de educação objetivando conscientizar as populações para a defesa e preservação do umbuzeiro, podendo celebrar convênios com organizações da sociedade civil, respeitadas as realidades de cada região, prevendo a pena de detenção de três meses a um ano e multa a quem a descumprir.

A legislação ambiental brasileira é uma das mais completas do mundo (Freiria, 2015), nesse sentido, a Instrução Normativa IBAMA no 191/2008 (IBAMA, 2008), a Resolução CEPRAN nº 1.009/1994 (CEPRAN, 1994) e o Projeto de Lei no 3.548B (Brasil, 2004) são instrumentos fundamentais para proteção da biodiversidade brasileira. No entanto é preciso que as agencias reguladoras e fiscalizadoras tenham condições humanas e materiais de aplicação da legislação, coibindo as ações que possam promover impactos sobre a biodiversidade (Ferro et al., 2006). 
Tabela 2. Lista de espécies da flora registradas para a área de mineração do gnaisse na Comunidade Vila Renascer em Petrolina, Pernambuco Nordeste do Brasil.

\begin{tabular}{|c|c|c|c|}
\hline Táxon & $\begin{array}{c}\text { Nome } \\
\text { popular }\end{array}$ & $\begin{array}{l}\text { Endemismo na } \\
\text { caatinga }\end{array}$ & Status de conservação \\
\hline \multicolumn{4}{|l|}{ Fabaceae } \\
\hline $\begin{array}{l}\text { Poincianella pyramidalis (Tul.) E. } \\
\text { Gagnon \& G. P. Lewis }\end{array}$ & Catingueira & - & NA \\
\hline Mimosa tenuiflora Benth. & Jurema-preta & - & NA \\
\hline $\begin{array}{l}\text { Anadenanthera colubrina (Vell.) } \\
\text { Brenan }\end{array}$ & Angico & - & NA \\
\hline $\begin{array}{l}\text { Hymenaea stigonocarpa Mart. ex } \\
\text { Hayne }\end{array}$ & Jatobá & $\mathrm{X}$ & NA \\
\hline $\begin{array}{l}\text { Senna trachypus (Benth.) H.S. } \\
\text { Irwin \& Barneby }\end{array}$ & Quebra-faca & - & NA \\
\hline $\begin{array}{l}\text { Amburana cearensis (Allemão) } \\
\text { A.C. Sm. }\end{array}$ & $\begin{array}{l}\text { Imburana-de- } \\
\text { cheiro }\end{array}$ & - & NA \\
\hline \multicolumn{4}{|l|}{ Anacardiaceae } \\
\hline Spondias tuberosa Arruda & Umbuzeiro & - & $\begin{array}{c}\text { Projeto de Lei } n^{\circ} \\
3.548 / 2004 \text { (PROT) }\end{array}$ \\
\hline Schinopsis brasiliensis Engl & Baraúna & - & $\begin{array}{c}\text { ENCEPRAM no } \\
1.009 / 1994(\text { PROT) }\end{array}$ \\
\hline Myracrodruon urundeuva Fr. All & Aroeira & - & $\begin{array}{c}\text { IUCN (LC); CEPRAM n } \\
1.009 / 1994 \text { (PROT) }\end{array}$ \\
\hline \multicolumn{4}{|l|}{ Burseraceae } \\
\hline $\begin{array}{l}\text { Commiphora lepthophloeos } \\
\text { (Mart.) J.B.Gillet }\end{array}$ & Imburana & - & NA \\
\hline \multicolumn{4}{|l|}{ Cactaceae } \\
\hline Cereus jamacaru D.C. & Mandacaru & $\mathrm{X}$ & IUCN (LC) \\
\hline $\begin{array}{l}\text { Pilosocereus gounellei (A. Weber } \\
\text { ex K. Schum.) Bly. ex Rowl.) }\end{array}$ & Xique-xique & $\mathrm{X}$ & NA \\
\hline $\begin{array}{l}\text { Melocactus bahiensis (Britton \& } \\
\text { Rose) Luetzelb. }\end{array}$ & Coroa de frade & $\mathrm{X}$ & $\mathrm{LC}$ \\
\hline \multicolumn{4}{|l|}{ Rhamnaceae } \\
\hline Ziziphus joazeiro Mart. & Juazeiro & $\mathrm{X}$ & NA \\
\hline \multicolumn{4}{|l|}{ Bromeliaceae } \\
\hline $\begin{array}{l}\text { Encholirium spectabile Mart. ex } \\
\text { Schult. \& Schult.f. }\end{array}$ & Macambira & $\mathrm{X}$ & NA \\
\hline \multicolumn{4}{|l|}{ Euphorbiaceae } \\
\hline Cnidoscolus quercifolius Pohl. & Favela & $\mathrm{X}$ & NA \\
\hline
\end{tabular}

Observação: Status de conservação de acordo com CNCFLORA (2013) e IUCN (2016); a Legislação: Instrução Normativa no 191/2008 (IBAMA, 2008); Resolução no 1.009/1994 (CEPRAN, 1994) e o Projeto de Lei no 3.548B (Brasil, 2004). Legenda: LC: Menos preocupante; NA - Não avaliada; PROT: Espécie Protegida.

\section{Conclusões}

Diante do exposto, a paisagem como também toda a dinâmica do meio ambiente é alterada após a atividade da mineração neste espaço, pois a retirada da vegetação nativa vai causar a degradação dessas áreas, impactos na paisagem local, extinção de espécies da caatinga, transtornos na saúde da população e também depredação de patrimônio particular como as várias marcas nas estruturas das residências pelo uso de explosivos, 
assim acometendo a estrutura das residências com rachaduras, como descrito por Silva e et al. (2018a).

Ademais, de acordo com o art. 225, § 1ํ, inciso VII, da Constituição Federal de 1988 (Brasil, 1988), são expressamente vedadas as práticas que coloquem em perigo a função ecológica e deixem de proteger a fauna e a flora, promovendo a extinção de (Machado, 2011).

A extração de minérios e os objetivos de proteção da fauna e da flora, são processos de complexa compatibilidade. No entanto, o Estado brasileiro busca através da Política Nacional de Meio Ambiente, a prevenção e a mitigação dos impactos da mineração sobre o meio ambiente, realizado através do licenciamento ambiental, com base no planejamento dos empreendimentos, consubstanciados nos documentos denominados Relatório de Controle Ambiental (RCA), Plano de Controle Ambiental (PCA), Relatório Ambiental Preliminar (RAP), Estudo de Impacto Ambiental (EIA) e Relatório de Impacto Ambiental (RIMA) (Mechi e Sanches, 2010).

Além do licenciamento ambiental, temos o princípio do poluidor-pagador da compensação ambiental, entendido como um instrumento econômico de política ambiental que exige do poluidor ou de potenciais poluidores o dever de arcar com as despesas estatais relativas à prevenção, reparação ou repressão dos danos ambientais (Araújo, 2011).

Destarte, incentivar o pagamento por serviços ambientais é uma forma de se alcançar o tão desejado desenvolvimento sustentável, nos dias de hoje, entretanto, é preciso efetuar a atualização das normas sobre o ICMS ecológico, garantindo, dessa forma, sua aplicabilidade e se instituir o mercado de carbono em atendimento ao que dispõe o novo Código Florestal (Araújo, 2011), temas estes que devem nortear e ampliar debates futuros sobre os impactos da mineração na biodiversidade, atrelado a estudos que discutam a capacidade de resiliência da biodiversidade (Oliveira, 2018), em ambientes que sofreram grandes alterações.

\section{Agradecimentos}

Os autores agradecem a bolsa de mestrado - Demanda Social da Coordenação de Aperfeiçoamento de Pessoal de Nível Superior (CAPES) direcionada ao primeiro autor no período do mestrado. Como também aos moradores da Comunidade da Vila Renascer em Petrolina, Pernambuco.

\section{Conflito de interesses}

Os autores declaram não haver conflito de interesses.

\section{Referências}

Albuquerque, U. P.; Cunha, L. V. F. C.; Lucena, R. F. P.; Alves, R. R. N. Methods and tecniques in Ethnobiology and Ethnoecology. New York: Humana Press, 2014.

Alves, J. J.; Araújo, M. A.; Nascimento, S. S. Degradação da caatinga: uma investigação ecogeográfica. Caminhos de Geografia, v. 9, n. 27, p. 143-155, 2008.

Alves, R. R. N.; Gonçalves, M. B. R.; Vieira, W. L. S. Caça, uso e conservação de vertebrados no semiárido Brasileiro. Tropical Conservation Science, v. 5, n.3 p. 94-416, 2012. https://doi.org/10.1177/194008291200500312 
Araújo, D. M. Os dilemas do princípio do poluidor-pagador na atualidade. Planeta Amazônia: Revista Internacional de Direito Ambiental e Políticas Públicas, n. 3, p. 153-162, 2011.

Bacci, D. D. L. C.; Landim, P. M. B.; Eston, S. M. Aspectos e impactos ambientais de pedreira em área urbana. REM: Revista Escola de Minas, v. 59, n. 1, p. 47-54, 2006. https://doi.org/10.1590/S0370-44672006000100007

Brasil. Constituição da República Federativa do Brasil de 1988. Disponível em: <http://www.planalto.gov.br/ccivil_03/constituicao/constituicao.htm>. Acesso em: 12 jul. 2020.

Brasil. Lei no 6.938, de 31 de agosto de 1981. Dispõe sobre a Política Nacional do Meio Ambiente, seus fins e mecanismos de formulação e aplicação, e dá outras providências. Disponível em: <http://www.planalto.gov.br/ccivil_03/leis/L6938compilada.htm>. Acesso em: 12 jul. 2020.

Brasil. Lei no 9.605, 12 de fevereiro de 1998. Dispõe sobre as sanções penais e administrativas derivadas de condutas e atividades lesivas ao meio ambiente, e dá outras providências. Disponível em: <http://www.planalto.gov.br/ccivil_03/leis/ 19605.htm>. Acesso em: 12 jul. 2020.

Brasil. Câmara dos Deputados, Coordenação de Comissões Permanentes. Projeto de Lei no 3.548-B, 2004. Disponível em: <https://www.camara.leg.br/proposicoesWeb/ prop_mostrarintegra;jsessionid=0F6BC6DAA4E881BA336A41571491BC7C.node2?codteo r=361064\&filename=Avulso+-PL+3548/2004>. Acesso em: 12 jul. 2020.

CEPRAM - Conselho Estadual do Meio Ambiente. Resolução no 1.009, de 6 de dezembro de 1994. Dispõe sobre proibição do corte, armazenamento e comercialização das espécies nativas, "aroeira" - Astronium urundeuva (Fr. Ali) Eng., "baraúna" - Schinopsis braslliensis Eng. e "Angico" - Anadenanthera macrocarpa (Benth) Brenan, no Estado da Bahia. Disponível em: <http://www.seia.ba.gov.br/legislacao-ambiental/resolucoes/resolu-ocepram-n-1009>. Acesso em: 12 jul. 2020.

CNCFLORA - Centro Nacional de Conservação da Flora. Livro Vermelho da Flora do Brasil. 1. ed. Rio de Janeiro: Andrea Jakobsson: Instituto de Pesquisas Jardim Botânico do Rio de Janeiro, 2013.

Drummond, G. M.; Martins, C. S.; Machado, A. B. M.; Sebaio, F. A.; Antonini, Y. Biodiversidade em Minas Gerais: um atlas para sua conservação. 2. ed. Belo Horizonte: Fundação Biodiversitas, 2005.

Faleiro, F. F.; Lopes, L. M. Aspectos da mineração e impactos da exploração de quartzito em Pirenópolis-GO. Ateliê Geográfico, v. 4, n. 3, p. 148-162, 2010. https://doi.org/10.5216/ ag.v4i3.16655

Ferro, A. F. P.; Bonacelli, M. B. M.; Assad, A. L. D. Oportunidades tecnológicas e estratégias concorrenciais de gestão ambiental: o uso sustentável da biodiversidade brasileira. Gestão \& Produção, v. 13, n. 3, p. 489-501, 2006. https://doi.org/10.1590/S0104530X2006000300011

Freiria, R. C. Aspectos históricos da legislação ambiental no Brasil: da ocupação e exploração territorial ao desafio da sustentabilidade. História e Cultura, v. 4, n. 3, p. 157-179, 2015.

Haddaway, N. R.; Cooke, S. J.; Lesser, P.; Macura, B.; Nilsson, A. E.; Taylor, J. J.; Raito, K. Evidence of the impacts of metal mining and the effectiveness of mining mitigation measures on social-ecological systems in Arctic and Boreal Regions: A systematic map

Rev. Bras. Gest. Amb. Sustent., 2020, vol. 7, n. 17, p. 1471-1482. 
protocol. Environmental Evidence, v. 8, Article number 9, 2019. https://doi.org/ 10.1186/s13750-019-0152-8

IBAMA - Instituto Brasileiro do Meio Ambiente e dos Recursos Naturais Renováveis. Instrução Normativa no 191, de 24 de setembro de 2008. Proíbe o corte do licuri (Syagrus coronata (Mart) Becc)) nas áreas de ocorrência natural desta palmeira nos Estados que especifica, até que sejam estabelecidas normas de manejo da espécie. Diposnível em: <https://www.normasbrasil.com.br/norma/instrucao-normativa-1912008_76905.html>. Acesso em: 12 jul. 2020.

ICMBio - Instituto Chico Mendes de Conservação da Biodiversidade. Livro Vermelho da Fauna Brasileira Ameaçada de Extinção. 1. ed. Brasília: ICMBio/MMA, 2018. v. 1.

IUCN - International Union for Conservation of Nature's. Lista vermelha da União Mundial para Conservação da Natureza. 2016. Disponível em: <https://www.iucnredlist.org>. Acesso em: 20 jul. 2020.

Lèvêque, C. A biodiversidade. Bauru: EDUSC, 1999.

Machado, P. A. L. Direito Ambiental brasileiro. 19 ed. São Paulo: Malheiros, 2011.

Mechi, A.; Sanches, D. L. Impactos ambientais da mineração no Estado de São Paulo. Estudos Avançados, v. 24, n. 68, p. 209-220, 2010. https://doi.org/10.1590/S010340142010000100016.

Oliveira, L. N. Análise da capacidade de resiliência do ambiente na área do baixo curso da Bacia Hidrográfica do Rio Poti (Piauí). Recife: Universidade Federal de Pernambuco, 2018. (Tese de doutorado).

Oliveira, J. A.; Gonçalves, P. R.; Bonvicino, C. R. Mamíferos da Caatinga. In: Leal, I. R.; Tabarelli, M.; Silva, J. M. C. Ecologia e conservação da Caatinga. Recife: Ed. UFPE, 2003. p. 275-335.

Pacheco, C. S. G. R.; Santos, R. P. Mining and its impacts on the "Caatingas" of the Brazilian Semiárido. International Journal of Advanced Engineering Research and Science, v. 6, n. 5, p. 178-189, 2019.

Peccatiello, A. F. O. Políticas públicas ambientais no Brasil: da administração dos recursos naturais (1930) à criação do Sistema Nacional de Unidades de Conservação (2000). Desenvolvimento e Meio Ambiente, n. 24, p.71-82, 2011. https://doi.org/10.5380/ dma.v24i0.21542

Pena, J. C. C.; Goulart, F.; Fernandes, G. W.; Hoffmann, D.; Leite, F. S. F.; Santos, N. B.; Soares Filho, B.; Souza, T. S.; Vancine, H.; Rodrigues, M. Impacts of mining activities on the potential geographic distribution of Eastern Brazil mountaintop endemic species. Perspectives in Ecology and Conservation, v. 15, p.172-178, 2017. https://doi.org/10.1016/j.pecon.2017.07.005

Pereira, J. A. A.; Borges L. A. C.; Barbosa, A. C. M. C.; Borem, R. A. T. Fundamentos da avaliação de impactos ambientais com estudo de caso. Lavras: UFLA, 2014.

Pires Junior, J.; Feio. R.; Luz, S.; Assis, C.; Guedes, J. J. M; Oliveira, L. A.; Lorenção, G. S. Herpetofauna em área de mineração de bauxita em São Sebastião da Vargem Alegre, Zona da Mata, Minas Gerais, Brasil. Anais do IX Congresso Brasileiro de Herpetologia, Campinas, Galoá, 2019. Disponível em: <https://proceedings.science/cbh2019/papers/herpetofauna-em-area-de-mineracao-de-bauxita-em-sao-sebastiao-davargem-alegre--zona-da-mata--minas-gerais--brasil>. Acesso em: 12 jul. 2020. 
Pontes, J. C.; Farias, M. S. S.; Lima, V. L. A. Mineração e seus reflexos socioambientais: estudo de impactos de vizinhança (EIV) causados pelo desmonte de rochas com uso de explosivos. Polêm!ca, v. 12, $\quad$ n. 1, p. 77-90, 2013. https://doi.org/10.12957/ polemica.2013.5277

Roos, A. A biodiversidade e a extinção das espécies. Revista Eletrônica em Gestão, Educação e Tecnologia Ambiental, v. 7, n. 7, p. 1494-1499, 2012.

Rudke, A. P.; Souza, V. A. S.; Santos, A. M.; Xavier, A. C. F.; Rotunno Filho, O. C.; Martins, J. A. Impact of mining activities on areas of environmental protection in the southwest of the Amazon: A GIS- and remote sensing-based assessment. Journal of Environmental Management, v. 263, 2020. https://doi.org/10.1016/j.jenvman.2020.110392

Santos, C. A. B.; Albuquerque, U. P.; Souto, W. M. S.; Alves, R. R. N. Assessing the effects of indigenous migration on zootherapeutic practices in the Semiarid Region of Brazil. PLoS ONE, v. 11, n. 1, p. 1-14, 2016. https://doi.org/10.1371/journal.pone.0146657

Silva, F. P.; Moura, G. J. B.; Santos, C. A. B. Representações dos moradores do entorno das áreas de exploração sobre a importância e impactos da mineração. Geosul, v. 33, n. 66, p. 128-146, 2018a. https://doi.org/10.5007/2177-5230.2018v33n66p128

Silva, F. P.; Rodrigues, M. S.; Moura, G. J. B.; Santos, C. A. B. Produção do espaço pela exploração do gnaisse na comunidade da Vila Renascer em Petrolina-PE. Geoambiente On-Line, n. 32, p. 96-113, 2018b. https://doi.org/10.5216/revgeoamb.v0i32.51639

Terborgh, J.; Schaik, C. V. Por que o mundo necessita de parques? In: Terborgh, J.; Schaik, C.; Davenport, L.; Rao, M. (Orgs.). Tornando os parques eficientes: estratégias para conservação da natureza nos trópicos. Curitiba: UFPR, 2002. p. 25-36.

Viana, M. B. Panorama do setor mineral: legislação e impactos socioambientais. Brasília: Câmara dos Deputados, 2015.

Wahsha, M.; Maleci, L.; Bini, C. The impact of former mining activity on soils and plants in the vicinity of an old mercury mine (Vallalta, Belluno, NE Italy). Geochemistry: Exploration, Environment, Analysis, v. 19, n. 2, p. 171-175, 2019. https://doi.org/ 10.1144/geochem2018-040

Wilson, S. A. Mining-induced displacement and resettlement: The case of rutile mining communities in Sierra Leone. Journal of Sustainable Mining, v. 18, p. 67-76, 2019. https://doi.org/10.1016/j.jsm.2019.03.001

Wu, Z.; Lei, S.; Lu, Q.; Bian, Z.; Ge, S. Spatial distribution of the impact of surface mining on the landscape ecological health of semi-arid grassland. Ecological Indicators, v. 111, 2020. https://doi.org/10.1016/j.ecolind.2019.105996

Informação da Licença: Este é um artigo Open Access distribuído sob os termos da Licença Creative Commons Attribution, que permite uso irrestrito, distribuição e reprodução em qualquer meio, desde que a obra original seja devidamente citada. 